PROCEEDINGS OF THE

AMERICAN MATHEMATICAL SOCIETY

Volume 130, Number 6, Pages 1755-1764

S 0002-9939(01)06259-1

Article electronically published on October 24, 2001

\title{
ON THE DISTRIBUTION SINGULAR VALUES OF TOEPLITZ MATRICES
}

\author{
MILUTIN R. DOSTANIĆ
}

(Communicated by David R. Larson)

\begin{abstract}
We prove a second order formula concerning distribution of singular values of Toeplitz matrices in some cases when conditions of the H. Widom Theorem are not satisfied.
\end{abstract}

\section{INTRODUCTION AND NOTATION}

In 1920 G. Szegö proved a basic result concerning the distribution of the eigenvalues $\left\{\lambda_{k}^{(n)}\right\}_{k=1}^{n}$ of the Toeplitz matrix

$$
T_{n}(f)=\left(\hat{f}_{i-j}\right)_{i, j=0}^{n-1}\left(\hat{f}_{k}=\frac{1}{2 \pi} \int_{-\pi}^{\pi} f(\theta) e^{-i k \theta} d \theta\right)
$$

associated with a bounded real valued function $f$ on the interval $[-\pi, \pi]$ : For any continuous function $F$ one has

$$
\lim _{n \rightarrow \infty} \frac{\sum_{k=1}^{n} F\left(\lambda_{k}^{(n)}\right)}{n}=\frac{1}{2 \pi} \int_{-\pi}^{\pi} F(f(\theta)) d \theta .
$$

An analogous result holds for the singular values $s_{1}^{(n)} \geq s_{2}^{(n)} \geq \ldots \geq s_{n}^{(n)}$ of not necessarily selfadjoint Toeplitz matrices $T_{n}(f)$. The analogue of (1) is

$$
\lim _{n \rightarrow \infty} \frac{\sum_{k=1}^{n} F\left(s_{k}^{(n)}\right)}{n}=\frac{1}{2 \pi} \int_{-\pi}^{\pi} F(|f(\theta)|) d \theta .
$$

Let

$$
\begin{gathered}
K=\left\{f \in L^{\infty}(-\pi, \pi): \sum_{k=-\infty}^{\infty}|k|\left|\hat{f}_{k}\right|^{2}<\infty\right\}, \\
M=\|f\|_{\infty}, m=\operatorname{dist}(0, \operatorname{conv} R(f))
\end{gathered}
$$

where $R(f)$ denotes the essential range of $f$ and "conv" denotes the convex hull. For $f \in K$ we let

$$
\|f\|_{K}=\left(\sum_{k \in \mathbb{Z}}|k|\left|\hat{f}_{k}\right|^{2}\right)^{\frac{1}{2}} .
$$

Received by the editors April 26, 2000 and, in revised form, December 15, 2000.

2000 Mathematics Subject Classification. Primary 47B10, 47B35.

Key words and phrases. Toeplitz matrices, singular values. 
Then it is easy to see that each singular value of $T_{n}(f)$ belongs to the interval $[m, M]$ (see Lemma 1.2 in [7]).

Let $t_{k}^{(n)}=\left(s_{k}^{(n)}\right)^{2}$. H. Widom [7] proved a more exact formula than (11) and (2). Namely he proved

Theorem 1. If $f \in K$ and $G \in C^{3}\left[m^{2}, M^{2}\right]$, then

$$
\begin{aligned}
& \lim _{n \rightarrow \infty}\left(\sum_{k=1}^{n} G\left(t_{k}^{(n)}\right)-\frac{n}{2 \pi} \int_{-\pi}^{\pi} G\left(|f(\theta)|^{2}\right) d \theta\right) \\
& =\operatorname{tr}\left(G(T(\bar{f}) T(f))+G(T(f) T(\bar{f}))-2 T\left(G\left(|f|^{2}\right)\right)\right) .
\end{aligned}
$$

We denote by $T(f)$ the infinite Toeplitz matrix $\left(\hat{f}_{i-j}\right)_{i, j=0}^{\infty}$ and by $H(f)=$ $\left(\hat{f}_{i+j+1}\right)_{i, j=0}^{\infty}$ the infinite Hankel matrix associated with the symbol $f$.

If $f \in K$, it is obvious that $H(f)$ is a Hilbert-Schmidt operator. Moreover, $|H(f)|_{2} \leq\|f\|_{K}$, where $|\cdot|_{2}$ denotes the Hilbert-Schmidt norm of the operator $H(f)$ acting on $l^{2}$ of the nonnegative integers. For the little bit of the theory of trace class (i.e., nuclear) and the Hilbert-Schmidt operators that will be needed we refer the reader to [3].

It is easy to see that $T(\bar{f})=(T(f))^{*}$ and hence the operators $T(\bar{f}) T(f)$ and $T(f) T(\bar{f})$ are selfadjoint. Therefore, operators $G(T(\bar{f}) T(f))$ and $G(T(f) T(\bar{f}))$ are defined by the spectral theorem. If $f \geq 0$, the operator $T(f)$ is obviously nonnegative.

In the case of eigenvalues, formulae similar to (3) are established in [1] and [4] but under much more restrictive assumptions on $G$ (which is assumed to be analytic) and on the symbol $f$.

The function $G$ in Theorem 1 is given in terms of the function $F$ in relation (2) by $G(\lambda)=F(\sqrt{\lambda})$. For $G$ to belong to $C^{3}$ it is not enough that $F$ belongs to $C^{3}$ but we also must have $F^{\prime}(0)=F^{\prime \prime}(0)=F^{\prime \prime \prime}(0)=0$ (in the case when $m=0$ ).

It is conjectured in [7] that the condition $F \in C^{3}[0, M], F^{\prime}(0)=0$ (in the case $m=0)$ is sufficient for the statement of Theorem 1.

Essentially, in the case $m=0$ it is necessary to prove Theorem 1 when $F(\lambda)=$ $\lambda^{\beta}$ and $\beta$ small enough. In this paper, we shall prove formula (3) in the case $m=0$ and when the conditions of Theorem 1 are not satisfied.

\section{RESULT}

Theorem 2. Let $f \in K$ and $m=0$. If $F(\lambda)=\lambda^{\alpha}(\alpha \geq 2)$, or $F \in C^{6}[0, M]$, $F^{\prime}(0)=0$, then the operator

$$
S=F(\sqrt{T(\bar{f}) T(f)})+F(\sqrt{T(f) T(\bar{f})})-2 T(F(|f|))
$$

is of the trace class and

$$
\lim _{n \rightarrow \infty}\left(\sum_{k=1}^{n} F\left(s_{k}^{(n)}\right)-\frac{n}{2 \pi} \int_{-\pi}^{\pi} F(|f(\theta)|) d \theta\right)=\operatorname{tr} S .
$$

Remark 1. Theorem 2 is stated for the case $m=0$. If $m>0$, (4) holds according to Theorem 1 (Of course, in that case the condition $F^{\prime}(0)=0$ is superficial.) 
In the proof of Theorem 2 we will use the following Lemma of Lizorkin [5]:

Lemma 1. Let $\alpha \geq 1, \sigma>0$. Then

$$
(i \lambda)^{\alpha}=A \exp \left(-i \frac{\pi \alpha \lambda}{2 \cdot \sigma}\right) \int_{-\infty}^{\infty} e^{-i(\lambda-\sigma) \xi} d \varrho(\xi) \quad(|\lambda| \leq \sigma)
$$

where $\varrho$ is a nondecreasing function of bounded variation and $A=\exp (-i \pi \alpha)$, $\varrho(\xi)=\sum_{k<\frac{\xi \sigma}{\pi}} a_{k}$, where $a_{k}$ are positive numbers related to Fourier coefficients $c_{n}$ of the function $(i \lambda)^{\alpha} e^{i \lambda \frac{\pi \alpha}{2 \sigma}}$ by

$$
c_{k}=\bar{A} a_{k} e^{-i k \pi}
$$

Here the function $(i \lambda)^{\alpha} e^{i \lambda \frac{\pi \alpha}{2 \sigma}}$ is assumed to be periodically extended from $(-\sigma, \sigma)$ to the entire real line. Our convention is $z^{\gamma}=e^{\gamma \ln z}, \ln z=\ln |z|+i \arg z$, $0 \leq \arg z<2 \pi$.

Lemma 2. If $1<\alpha<2$ and $M>0$, then there exists a nondecreasing function $\varrho_{0}$ of bounded variation, such that for each $\lambda \in\left[0, M^{2}\right]$

$$
\lambda^{\alpha}=\int_{-\infty}^{\infty} e^{-i 2 \pi \alpha-i M^{2} t} e^{i \lambda t} d \varrho_{0}(t)
$$

holds and $\int_{-\infty}^{\infty} t^{2} d \varrho_{0}(t)<\infty$.

Proof. We apply Lemma 1, with $\sigma=M^{2}$. Since $\lambda \geq 0$, we have $(i \lambda)^{\alpha}=e^{i \frac{\pi}{2} \alpha} \cdot \lambda^{\alpha}$ and thus

$$
\lambda^{\alpha} e^{-i \frac{\pi}{2} \alpha}=e^{-\pi \alpha} e^{i \lambda \frac{\pi \alpha}{2 M^{2}}} \int_{-\infty}^{\infty} e^{i\left(\lambda-M^{2}\right) \xi} d \varrho(\xi)
$$

i.e.,

$$
\lambda^{\alpha}=e^{-\frac{3 \pi i \alpha}{2}} \int_{-\infty}^{\infty} e^{-i M^{2} \xi} e^{i \lambda\left(\xi-\frac{\pi \alpha}{2 M^{2}}\right)} d \varrho(\xi)
$$

Substituting $\xi-\frac{\pi \alpha}{2 M^{2}}=t, \varrho_{0}(t) \stackrel{\text { def }}{=} \varrho\left(t+\frac{\pi \alpha}{2 M^{2}}\right)$ in the last formula we get

$$
\lambda^{\alpha}=\int_{-\infty}^{\infty} e^{-2 \pi i \alpha} e^{-i M^{2} t} e^{i \lambda t} d \varrho_{0}(t) .
$$

The last formula holds for $\lambda \in\left[0, M^{2}\right]$ and $\alpha \geq 1$. Since $\varrho$ is a function of bounded variation, so is $\varrho_{0}$.

We will show now that for $1<\alpha<2$,

$$
\int_{-\infty}^{\infty} t^{2} d \varrho_{0}(t)<\infty
$$

Since $\varrho_{0}$ is a function of bounded variation, applying the Cauchy inequality to (5) we get

$$
\int_{-\infty}^{\infty}|t| d \varrho_{0}(t)<\infty .
$$

Since $\varrho_{0}(t)=\varrho\left(t+\frac{\pi \alpha}{2 M^{2}}\right)$, in order to prove (15) it is enough to show that

$$
\int_{-\infty}^{\infty} t^{2} d \varrho(t)<\infty .
$$


From the way the function $\varrho$ is defined it follows that it suffices to prove

$$
\sum_{n \in \mathbb{Z}} n^{2} a_{n}<\infty
$$

where $a_{n}$ is the sequence of positive numbers from Lemma 1 (with $\sigma=M^{2}$ ), i.e., the convergence of the series

$$
\sum_{n \in \mathbb{Z}}(-1)^{n} n^{2} c_{n}
$$

where $c_{n}$ are the Fourier coefficients of the function $(i \lambda)^{\alpha} e^{i \lambda \frac{\pi \alpha}{2 M^{2}}}$ on the interval $\left[-M^{2}, M^{2}\right]$. Since

$$
\begin{gathered}
\int_{-M^{2}}^{M^{2}}(i \lambda)^{\alpha} \exp \left(\frac{i \lambda \pi \alpha}{2 M^{2}}\right) \cdot \exp \left(-\frac{i \lambda n \pi}{M^{2}}\right) d \lambda \\
=\left(\frac{M^{2}}{\pi}\right)^{\alpha+1} \cdot \int_{-\pi}^{\pi}(i x)^{\alpha} e^{\frac{i \alpha x}{2}} e^{-i n x} d x,
\end{gathered}
$$

in order to prove the convergence of the series (6) it is enough to prove that for $1<\alpha<2$ the series

$$
\sum_{n \in \mathbb{Z}}(-1)^{n} n^{2} \int_{-\pi}^{\pi}(i x)^{\alpha} e^{\frac{i \alpha x}{2}} e^{-i n x} d x
$$

converges.

Since $\alpha>1$, integrating by parts twice and having in mind the definition of the function $z \longmapsto z^{\gamma}$, we conclude that the convergence of the above series will be established once we prove that the series

$$
\sum_{n \in \mathbb{Z}}(-1)^{n} A_{n}
$$

converges for $1<\alpha<2$. Here $A_{n} \stackrel{\text { def }}{=} \int_{-\pi}^{\pi}(i x)^{\alpha-2} e^{\frac{i \alpha x}{2}} e^{-i n x} d x$. Consider now the behavior of $A_{n}$ as $n \rightarrow \infty$. If $n>0$, one gets

$$
\begin{aligned}
A_{n}=\left(n-\frac{\alpha}{2}\right)^{1-\alpha}\left[-e^{\frac{i \pi \alpha}{2}} \cdot \int_{0}^{\pi\left(n-\frac{\alpha}{2}\right)} t^{\alpha-2} e^{-i t} d t-e^{\frac{3 \pi i \alpha}{2}}\right. \\
\left.\cdot \int_{0}^{\pi\left(n-\frac{\alpha}{2}\right)} t^{\alpha-2} e^{i t} d t\right] .
\end{aligned}
$$

Since for $1<\alpha<2 \int_{0}^{\infty} t^{\alpha-2} e^{ \pm i t} d t=\Gamma(\alpha-1) e^{ \pm i \frac{\pi}{2}(\alpha-1)}$ we obtain

$$
\begin{array}{r}
A_{n}=\left(n-\frac{\alpha}{2}\right)^{1-\alpha}\left[i \Gamma(\alpha-1)\left(e^{2 \pi i \alpha}-1\right)+e^{\frac{i \pi \alpha}{2}} \cdot \int_{\pi\left(n-\frac{\alpha}{2}\right)}^{\infty} t^{\alpha-2} e^{-i t} d t\right. \\
\left.+e^{\frac{3 \pi i \alpha}{2}} \cdot \int_{\pi\left(n-\frac{\alpha}{2}\right)}^{\infty} t^{\alpha-2} e^{i t} d t\right] .
\end{array}
$$


Integrating by parts, we get

$$
\begin{aligned}
\int_{\pi\left(n-\frac{\alpha}{2}\right)}^{\infty} t^{\alpha-2} e^{-i t} d t & =(-1)^{n+1} i\left(\pi\left(n-\frac{\alpha}{2}\right)\right)^{\alpha-2} \cdot e^{i \frac{\pi \alpha}{2}}+O\left(n^{\alpha-3}\right), \\
\int_{\pi\left(n-\frac{\alpha}{2}\right)}^{\infty} t^{\alpha-2} e^{i t} d t & =(-1)^{n} i\left(\pi\left(n-\frac{\alpha}{2}\right)\right)^{\alpha-2} \cdot e^{-i \frac{\pi \alpha}{2}}+O\left(n^{\alpha-3}\right), \quad n \rightarrow \infty
\end{aligned}
$$

and thus,

$$
A_{n}=\left(n-\frac{\alpha}{2}\right)^{1-\alpha}\left[i \Gamma(\alpha-1)\left(e^{2 \pi i \alpha}-1\right)+O\left(n^{\alpha-3}\right)\right] .
$$

Therefore, the series $\sum_{n=1}^{\infty}(-1)^{n} A_{n}$ converge. In a similar way one shows that the series

$$
\sum_{n=-\infty}^{-1}(-1)^{n} A_{n}
$$

also converge.

Remark 2. From Lemma 2 (by integrating over $\lambda$ ) we obtain the representation

$$
\lambda^{\alpha+1}=\int_{-\infty}^{+\infty} e^{-2 \pi i \alpha-i M^{2} t} \cdot \frac{e^{i \lambda t}-1}{i t}(\alpha+1) d \rho_{0}(t),
$$

for $1<\alpha<2, \lambda \in\left[0, M^{2}\right]$.

Let $d \nu=\frac{\alpha+1}{i t} d \rho_{0}$. If $1<\alpha<2$, then the function $\rho_{0}$ does not have a jump at the $t=0$, hence

$$
\int_{-\infty}^{+\infty}|t|^{k} d|\nu|<\infty \quad \text { for } k=0,1,2,3
$$

$(|\nu|$ is a variation of measure $\nu)$. From (7), putting $\beta=\alpha+1$, we get

$$
\lambda^{\beta}=\int_{-\infty}^{+\infty} e^{-2 \pi i \alpha-i M^{2} t} e^{i \lambda t} d \nu(t)-A, \quad 2<\beta<3, \lambda \in\left[0, M^{2}\right],
$$

and

$$
A=\int_{-\infty}^{+\infty} e^{-2 \pi i \alpha-i M^{2} t} d \nu(t)
$$

We write $P_{n}$ for the projection operator, defined by

$$
P_{n}\left(x_{0}, x_{1} \ldots\right)=\left(x_{0}, x_{1}, x_{2}, \ldots, x_{n-1}, 0,0, \ldots\right)
$$

from $l^{2}$ to the subspace of $l^{2}$ on which $T_{n}(f)$ may be thought of as acting. We identify $T_{n}(f)$ with $P_{n} T(f) P_{n}$ in the obvious way. We define an operator $Q_{n}$ on $l^{2}$ by

$$
Q_{n}\left(x_{0}, x_{1}, \ldots\right)=\left(x_{n-1}, x_{n-2}, \ldots, x_{1}, x_{0}, 0,0, \ldots\right)
$$

For $f \in L^{\infty}(-\pi, \pi)$ we define $\tilde{f}(\theta)=f(-\theta)$. 
Lemma 3. 1) For any $f, g \in L^{\infty}(-\pi, \pi)$ we have

$$
\begin{aligned}
& T(f \cdot g)-T(f) \cdot T(g)=H(f) H(\tilde{g}), \\
& T_{n}(f \cdot g)-T_{n}(f) \cdot T_{n}(g)=P_{n} H(f) H(\tilde{g}) P_{n}+Q_{n} H(\tilde{f}) H(g) Q_{n} .
\end{aligned}
$$

2) If $f \in K$, then we have $\tilde{f} \in K,|f|^{2} \in K, e^{i t f} \in K(\forall t \in \mathbb{R})$ and

$$
\begin{aligned}
\left\|\left|f^{2}\right|\right\|_{K} & \leq \text { const }\|f\|_{K}, \\
\left\|e^{i t f}\right\|_{K} & \leq \text { const } \cdot|t| \cdot\|f\|_{K} .
\end{aligned}
$$

Proof. 1) Routine computation. (Or see [2], Propositions 2.7 and 3.6.)

2) Can be proved in a same way as Proposition 1 in [6].

Lemma 4. If $f \in K, 1<\alpha<2$, then the operator $\left(T\left(|f|^{2}\right)\right)^{\alpha}-T\left(|f|^{2 \alpha}\right)$ is nuclear.

Proof. Integrating the identity

$$
\begin{aligned}
& \frac{d}{d s}\left(T\left(e^{i s|f|^{2}}\right) e^{-i s T\left(|f|^{2}\right)}\right) \\
& \quad=\left[T\left(e^{i s|f|^{2}} \cdot i|f|^{2}\right)-T\left(e^{i s|f|^{2}}\right) T\left(i|f|^{2}\right)\right] e^{-i s T\left(|f|^{2}\right)}
\end{aligned}
$$

on the interval $[0, t]$ and multiplying the result by $e^{i t T\left(|f|^{2}\right)}$ on the right, we get

$$
T\left(e^{i t|f|^{2}}\right)-e^{i t T\left(|f|^{2}\right)}=\int_{0}^{t} H\left(e^{i s|f|^{2}}\right) \cdot H\left(i|\tilde{f}|^{2}\right) e^{i(t-s) T\left(|f|^{2}\right)} d s .
$$

Since $f \in K$, we have $e^{i s|f|^{2}} \in K, i|f|^{2} \in K$. Applying Lemma 3, formula (9) yields

$$
\left|T\left(e^{i t|f|^{2}}\right)-e^{i t T\left(|f|^{2}\right)}\right|_{1} \leq \int_{0}^{|t|}\left|H\left(e^{i s|f|^{2}}\right) \cdot H\left(i|\tilde{f}|^{2}\right)\right|_{1} d s
$$

for all $t \in \mathbb{R}$, since the operator $e^{i(t-s) T\left(|f|^{2}\right)}$ is unitary. Here $|\cdot|_{1}$ denotes the nuclear norm of an operator. Since the operators $H\left(e^{i s|f|^{2}}\right)$ and $H\left(i|\tilde{f}|^{2}\right)$ are HilbertSchmidt, their product is nuclear, and thus, according to Lemma 3 (statement 2)) we get

$$
\left|H\left(e^{i s|f|^{2}}\right) \cdot H\left(i|\tilde{f}|^{2}\right)\right|_{1} \leq c_{0} \cdot|s| \cdot\|f\|_{K}^{2}
$$

( $c_{0}$ is independent of $s$ ), and thus

$$
\left|T\left(e^{i t|f|^{2}}\right)-e^{i t T\left(|f|^{2}\right)}\right|_{1} \leq c_{0}\|f\|_{K}^{2} \int_{0}^{|t|} s d s=\frac{c_{0}}{2} t^{2}\|f\|_{K}^{2} .
$$

According to Lemma 2

$$
\begin{aligned}
\left(T\left(|f|^{2}\right)\right)^{\alpha} & =\int_{\mathbb{R}} e^{-2 \pi i \alpha-i M^{2} t} e^{i t T\left(|f|^{2}\right)} d \varrho_{0}(t), \\
|f|^{2 \alpha} & =\int_{\mathbb{R}} e^{-2 \pi i \alpha-i M^{2} t} e^{i t|f|^{2}} d \varrho_{0}(t),
\end{aligned}
$$

and thus,

$$
T\left(|f|^{2 \alpha}\right)=\int e^{-2 \pi i \alpha-i M^{2} t} T\left(e^{i t|f|^{2}}\right) d \varrho_{0}(t) .
$$


Therefore,

$$
\left(\left(T\left(|f|^{2}\right)\right)^{\alpha}-T\left(|f|^{2 \alpha}\right)\right)=\int_{\mathbb{R}} e^{-2 \pi i \alpha-i M^{2} t}\left(e^{i t T\left(|f|^{2}\right)}-T\left(e^{i t|f|^{2}}\right)\right) d \varrho_{0}(t) .
$$

Inequality (10) shows that the integral on the right side in the formula above, converges in nuclear norm and thus $\left(\left(T\left(|f|^{2}\right)\right)^{\alpha}-T\left(|f|^{2 \alpha}\right)\right)$ is a nuclear operator. Moreover,

$$
\left.\left|\left(\left(T\left(|f|^{2}\right)\right)^{\alpha}-T\left(|f|^{2 \alpha}\right)\right)\right|_{1} \leq \frac{c_{0}}{2}\|f\|_{K}^{2} \int_{\mathbb{R}} t^{2} d \varrho_{0}(t)<+\infty \quad \text { (by Lemma } 2\right)
$$

Lemma 5. If $1<\alpha<2$ and $f \in K$, then

$$
\lim _{n \rightarrow \infty} \operatorname{tr}\left(\left(T_{n}\left(|f|^{2}\right)\right)^{\alpha}-T_{n}\left(|f|^{2 \alpha}\right)\right)=2 \operatorname{tr}\left(\left(T\left(|f|^{2}\right)\right)^{\alpha}-T\left(|f|^{2 \alpha}\right)\right) .
$$

Proof. In a same way as we proved (9) we get

$$
\begin{aligned}
& T_{n}\left(e^{i t|f|^{2}}\right)-e^{i t T_{n}\left(|f|^{2}\right)} \\
& \quad=\int_{0}^{t}\left[T_{n}\left(e^{i s|f|^{2}} i|f|^{2}\right)-T_{n}\left(e^{i s|f|^{2}}\right) T_{n}\left(i|f|^{2}\right)\right] e^{i(t-s) T_{n}\left(|f|^{2}\right)} d s
\end{aligned}
$$

and thus by Lemma 3

$$
\begin{aligned}
T_{n}\left(e^{i t|f|^{2}}\right) & -e^{i t T_{n}\left(|f|^{2}\right)}=\int_{0}^{t}\left[P_{n} H\left(e^{i s|f|^{2}}\right) H\left(i|\tilde{f}|^{2}\right) P_{n}\right. \\
& \left.+Q_{n} H\left(e^{i s|\tilde{f}|^{2}}\right) H\left(i|f|^{2}\right) Q_{n}\right] e^{i(t-s) T_{n}\left(|f|^{2}\right)} d s .
\end{aligned}
$$

From Lemma 2 we obtain

$$
\begin{aligned}
& \left(\left(T_{n}\left(|f|^{2}\right)\right)^{\alpha}-T_{n}\left(|f|^{2 \alpha}\right)\right) \\
& \quad=\int_{\mathbb{R}} e^{-2 \pi i \alpha-i M^{2} t}\left(e^{i t T_{n}\left(|f|^{2}\right)}-T_{n}\left(e^{i t|f|^{2}}\right)\right) d \varrho_{0}(t) \quad(1<\alpha<2) .
\end{aligned}
$$

It follows from (11) that

$$
\left|T_{n}\left(e^{i t|f|^{2}}\right)-e^{i t T_{n}\left(|f|^{2}\right)}\right|_{1} \leq \mathrm{const}|t|^{2}, \quad \forall t \in \mathbb{R} .
$$

(const does not depend on $t$ and $n$ ) and thus, since $\int_{\mathbb{R}}|t|^{2} d \varrho_{0}(t)<\infty$, by the same arguments as in the proof of (14) in [7] and by the Lebesgue theorem on dominant convergence, (11) and (12) give

$$
\lim _{n \rightarrow \infty} \operatorname{tr}\left(\left(T_{n}\left(|f|^{2}\right)\right)^{\alpha}-T_{n}\left(|f|^{2 \alpha}\right)\right)=2 \operatorname{tr}\left(\left(T\left(|f|^{2}\right)\right)^{\alpha}-T\left(|f|^{2 \alpha}\right)\right) .
$$

Lemma 6. If $f \in K, 1<\alpha<2$, then the operator $(T(\bar{f}) T(f))^{\alpha}+(T(f) T(\bar{f}))^{\alpha}-$ $2 T\left(|f|^{2 \alpha}\right)$ is nuclear and

$$
\begin{aligned}
\lim _{n \rightarrow \infty} \operatorname{tr}\left[\left(T_{n}(\bar{f}) T_{n}(f)\right)^{\alpha}-T_{n}\left(|f|^{2 \alpha}\right)\right] \\
=\operatorname{tr}\left[(T(\bar{f}) T(f))^{\alpha}+(T(f) T(\bar{f}))^{\alpha}-2 T\left(|f|^{2 \alpha}\right)\right] .
\end{aligned}
$$


Proof. By Lemma 2$]$ we have

$$
\begin{aligned}
& (T(\bar{f}) T(f))^{\alpha}-T_{n}\left(|f|^{2}\right)^{\alpha} \\
& =\int_{-\infty}^{\infty} e^{-2 \pi i \alpha-i M^{2} t} \cdot\left(e^{i t T(\bar{f}) T(f)}-e^{i t T\left(|f|^{2}\right)}\right) d \varrho_{0}(t) .
\end{aligned}
$$

Since $\left|e^{i t T(\bar{f}) T(f)}-e^{i t T\left(|f|^{2}\right)}\right|_{1} \leq$ const $\cdot|t|(t \in \mathbb{R})$, and $\int_{\mathbb{R}}|t| d \varrho_{0}(t)<+\infty$, the integral in (13) converges (in nuclear norm) and thus the operator $(T(\bar{f}) T(f))^{\alpha}-$ $T\left(|f|^{2}\right)^{\alpha}$ is nuclear. In a similar way we prove that the operator $(T(f) T(\bar{f}))^{\alpha}-$ $T_{n}\left(|f|^{2}\right)^{\alpha}$ is nuclear. Thus $(T(\bar{f}) T(f))^{\alpha}+(T(f) T(\bar{f}))^{\alpha}-2 T\left(|f|^{2}\right)^{\alpha}$ is also nu-

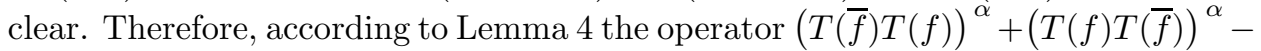
$2 T\left(|f|^{2 \alpha}\right)$ is nuclear. Since $\left|e^{i t T_{n}(\bar{f}) T(f)}-e^{i t T_{n}\left(|f|^{2}\right)}\right|_{1} \leq d_{0} \cdot|t|\left(t \in \mathbb{R}, d_{0}\right.$ is independent of $n$ and $t$ ) and $\int_{-\infty}^{\infty}|t| d \varrho_{0}(t)<+\infty$, it follows from

$$
\begin{aligned}
& \left(T_{n}(\bar{f}) T_{n}(f)\right)^{\alpha}-T_{n}\left(|f|^{2}\right)^{\alpha} \\
& \quad=\int_{-\infty}^{\infty} e^{-2 \pi i \alpha-i M^{2} t}\left(e^{i t T_{n}(\bar{f}) T(f)}-e^{i t T_{n}\left(|f|^{2}\right)}\right) d \varrho_{0}(t)
\end{aligned}
$$

that

$$
\begin{aligned}
& \lim _{n \rightarrow \infty} \operatorname{tr}\left(\left(T_{n}(\bar{f}) T_{n}(f)\right)^{\alpha}-T_{n}\left(|f|^{2}\right)^{\alpha}\right) \\
& \quad=\int_{-\infty}^{\infty} e^{-2 \pi i \alpha-i M^{2} t} \lim _{n \rightarrow \infty} \operatorname{tr}\left(e^{i t T_{n}(\bar{f}) T(f)}-e^{i t T_{n}\left(|f|^{2}\right)}\right) d \varrho_{0}(t) .
\end{aligned}
$$

From relation (14) in [7] and from Lemma 2, the last equality becomes

$$
\begin{aligned}
& \lim _{n \rightarrow \infty} \operatorname{tr}\left((T(\bar{f}) T(f))^{\alpha}-T_{n}\left(|f|^{2}\right)^{\alpha}\right) \\
& =\operatorname{tr}\left((T(\bar{f}) T(f))^{\alpha}+(T(f) T(\bar{f}))^{\alpha}-2 T\left(|f|^{2}\right)^{\alpha}\right) .
\end{aligned}
$$

From (14) and Lemma 5 adding, we obtain

$$
\begin{aligned}
& \lim _{n \rightarrow \infty} \operatorname{tr}\left[\left(T_{n}(\bar{f}) T_{n}(f)\right)^{\alpha}-T_{n}\left(|f|^{2 \alpha}\right)\right] \\
& =\operatorname{tr}\left[(T(\bar{f}) T(f))^{\alpha}+(T(f) T(\bar{f}))^{\alpha}-2 T\left(|f|^{2 \alpha}\right)\right] .
\end{aligned}
$$

Remark 3. By using representation (8) by the same method as the one used for proving Lemmas 4, 5, 6 one can show that:

Lemma 7. If $f \in K$ and $2<\beta<3$, then the operator $T\left(|f|^{2}\right)^{\beta}-T\left(|f|^{2 \beta}\right)$ is the trace class and the following holds:

$$
\lim _{n \longrightarrow \infty} \operatorname{tr}\left[T_{n}\left(|f|^{2}\right)^{\beta}-T_{n}\left(|f|^{2 \beta}\right)\right]=2 \operatorname{tr}\left[T\left(|f|^{2}\right)^{\beta}-T\left(|f|^{2 \beta}\right)\right] .
$$

Also, The operator $(T(\bar{f}) T(f))^{\beta}+(T(f) T(\bar{f}))^{\beta}-2 T\left(|f|^{2 \beta}\right)$ is the trace class and the following holds:

$$
\begin{aligned}
& \lim _{n \rightarrow \infty} \operatorname{tr}\left[\left(T_{n}(\bar{f}) T_{n}(f)\right)^{\beta}-T_{n}\left(|f|^{2 \beta}\right)\right] \\
& =\operatorname{tr}\left[(T(\bar{f}) T(f))^{\beta}+(T(f) T(\bar{f}))^{\beta}-2 T\left(|f|^{2 \beta}\right)\right] .
\end{aligned}
$$




\section{Proof of Theorem 2}

Note that Theorem 2 holds for the functions $\lambda \longmapsto \lambda^{2}, \lambda \longmapsto \lambda^{4}$ and $\lambda \longmapsto \lambda^{\alpha}$ $(\alpha \geq 6)$ as a consequence of Theorem 1. In other words,

$$
\begin{aligned}
& \lim _{n \rightarrow \infty}\left(\sum_{k=1}^{n}\left(s_{k}^{(n)}\right)^{\alpha}-\frac{n}{2 \pi} \int_{-\pi}^{\pi}|f(\theta)|^{\alpha} d \theta\right) \\
& \quad=\operatorname{tr}\left[(T(\bar{f}) T(f))^{\frac{\alpha}{2}}+(T(f) T(\bar{f}))^{\frac{\alpha}{2}}-2 T\left(|f|^{\alpha}\right)\right],
\end{aligned}
$$

for $\alpha=2,4$ and $\alpha \geq 6$.

From Lemma 6 and Lemma 7 we obtain that (15) also holds if $2<\alpha<4$ and $4<\alpha<6$. Therefore, formula (44) holds if $F(\lambda)=\lambda^{\alpha}$ and $\alpha \geq 2$.

Now let $F \in C^{6}\left[0, M^{2}\right]$ and $F^{\prime}(0)=0$. Then, for the function $F_{0}(\lambda)=$ $\sum_{k=2}^{6} \frac{F^{(k)}(0)}{k !} \lambda^{k}$ we have

$$
\begin{aligned}
& \lim _{n \rightarrow \infty}\left(\sum_{k=1}^{n} F_{0}\left(s_{k}^{(n)}\right)-\frac{n}{2 \pi} \int_{-\pi}^{\pi} F_{0}(|f(\theta)|) d \theta\right) \\
& =\operatorname{tr}\left[F_{0}(\sqrt{T(\bar{f}) T(f)})+F_{0}(\sqrt{T(f) T(\bar{f})})-2 T\left(F_{0}(|f|)\right)\right] .
\end{aligned}
$$

Let $R(\lambda)=F(\lambda)-F_{0}(\lambda)$. The function $\lambda \longmapsto R(\sqrt{\lambda})$ satisfies the conditions of Theorem 1 and hence

$$
\begin{aligned}
& \lim _{n \rightarrow \infty}\left(\sum_{k=1}^{n} R\left(s_{k}^{(n)}\right)-\frac{n}{2 \pi} \int_{-\pi}^{\pi} R(|f(\theta)|) d \theta\right) \\
& =\operatorname{tr}[R(\sqrt{T(\bar{f}) T(f)})+R(\sqrt{T(f) T(\bar{f})})-2 T(R(|f|))] .
\end{aligned}
$$

Adding (16) and (17) one gets (4). (The operators on the right-hand side of (16) and (17) are nuclear and so is their sum, i.e., the operator $S$ is nuclear.)

Remark 4. The question of whether the condition $F^{\prime}(0)=0$ in Theorem 2 is necessary remains open. To answer it affirmatively it is enough to find the example of a function $f \in K$ such that $m=\operatorname{dist}(0, \operatorname{conv} R(f))=0$ and

$$
\begin{aligned}
\lim _{n \rightarrow \infty}( & \sum_{k=1}^{n} s_{k}^{(n)}-\frac{n}{2 \pi} \int_{-\pi}^{\pi}|f(\theta)| d \theta \\
& -\operatorname{tr}[\sqrt{T(\bar{f}) T(f)}+\sqrt{T(f) T(\bar{f})}-2 T(|f|)]) \neq 0 .
\end{aligned}
$$

\section{ACKNOWLEDGMENT}

The author is grateful to the referee for the suggestion which helped to improve this article.

\section{REFERENCES}

[1] E.L. Basor, "Trace formulas for Toeplitz Matrices with Piecewise continuous Symbols", Journal of Mathematical Analysis and Applications, 120 (1986), 25-38. MR 88a:47027

[2] A. Bötcher and B. Silberman, "Invertibility and Asymptotics of Toeplitz Matrices", Berlin, Akademie-Verlag 1983. MR 85g:47037 
[3] I. C. Gohberg and M. G. Krein, "Introduction to the theory of linear nonselfadjoint operators", Translation of the Math. Monographs, Vol. 18, Amer. Math. Soc., Providence, R.I, 1969. MR 39:7447

[4] L.M. Libkind, "The asymptotic behavior of the eigenvalues of Toeplitz forms", Math. Notes, 11 (1972), 97-101.

[5] P.I. Lizorkin, "Estimates of trigonometric integrals and the Bernstein inequality for fractional derivatives" Izv. Akad. Nauk SSSR, Ser. Mat. T29 (1965), 109-126.

[6] H. Widom "A trace formula for Wiener-Hopf operators" J. Operator Theory, 8 (1982), 279-298. MR 84e: 47038

[7] H. Widom, "On the singular values of Toeplitz matrices" Zeitschrift fur Analysis und Ihre Anwendungen, 8 (1989), no. 3, 221-229. MR 90k:47058

Matematicki Fakultet, University of Belgrade, Studentski trg 16, 11000 Belgrade, YugOSLAVIA

E-mail address: domi@matf.bg.ac.yu 